REVIEW ARTICLE

\title{
Endothelial Function for Cardiovascular Disease Prevention and Management
}

\author{
Minako Yamaoka-Tojo*
}

School of Allied Health Sciences, Kitasato University, Japan

*Corresponding author: Minako Yamaoka-Tojo, MD, PhD, FAHA, FACP, FJCC, School of Allied Health Sciences, Kitasato University, 1-15-1 Kitasato, Minami-ku, Sagamihara, 252-0373, Japan, Tel: +81-42-778-8111, Fax: +81-42-778-9696, E-mail:myamaoka@med.kitasato-u.ac.jp

\section{Introduction}

Endothelium is a layer of the endothelial cells lining to the lumen of blood vessels, lymphatic vessels, the heart and other organs. Vascular endothelium is considered to be the largest endocrine organ in human body. Its total weight is about $1.5 \mathrm{~kg}$, and it is responsible for maintaining homeostasis of the living body by exerting various functions. Cardiovascular disease is mainly induced by atherosclerosis, which is produced by vascular inflammation [1]. Vascular endothelial dysfunction is an early stage of the onset of atherosclerosis, which affects the progression and onset of cardiovascular disease [2]. In the presence of coronary risk factors, vascular endothelial cells are induced phenotypic changes and functional changes, reduce the production and bioactivity of Nitric Oxide (NO), and thereby give a signal to contract the blood vessels, cause inflammation, and thrombus formation. Vascular endothelial dysfunction is known to be an independent risk factor for the onset of cardiovascular events, with or without previous history of coronary artery disease $[3,4]$.

Classical "vascular endothelial dysfunction" refers to abnormal vasorelaxation response to vasoactive substances such as acetylcholine and bradykinin. In recent years, it has become widely known that vascular endothelial dysfunction is deeply involved in the pathophysiology of cardiovascular disease onset and progression, not only mere vasodilatory disorder but also vascular function as an endocrine organ for inflammatory control, immune response, and coagulation and hemostasis regulation. Therefore, "vascular endothelial dysfunc- tion" is considered to be more broad sense, including a wide range of functional disorders of endothelial cells related to systemic responses. Vascular endothelial cells are directly affected by blood circulation and its circulating substances. Therefore, vascular endothelial function decreases with high blood glucose and blood lipid due to ingestion of meal. However, this is merely a physiological response and it recovers in about 4 to 6 hours after a meal, so it is only a temporary decrease in vascular endothelial function. Meanwhile, chronic hyperglycemic conditions, excessive fluctuation of blood glucose in patients with diabetes, fasting hyperlipidemia and postprandial hyperlipidemia in patients with dyslipidemia decrease vascular endothelial function in fasting conditions. Notably, vascular endothelial function is decreased by smoking and lifestyle diseases such as hypertension, diabetes mellitus, dyslipidemia, oxidative stress excess state, inflammation, and/or sympathetic hyperactivity. In these conditions, vascular endothelial functions are decreased and atherosclerorsis progression is exacerbated (Figure 1). Vascular endothelial function of coronary arteries is known to be associated with myocardial ischemia $[5,6]$. In addition, vascular endothelial dysfunction is an early pro-arteriosclerotic change [7], and it is deeply related to an increase in fetal events in ischemic heart disease and cerebrovascular disease $[3,8-10]$.

\section{Importance of Total Risk Management for Preventive Cardiovascular Disease}

The main objective of the total risk management for atherosclerosis patients to prevent cardiovascular dis-

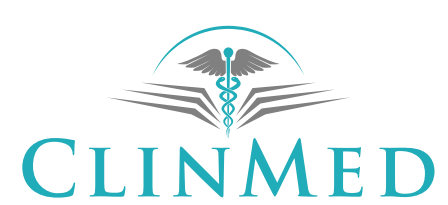

INTERNATIONAL LIBRARY
Citation: Yamaoka-Tojo M (2017) Endothelial Function for Cardiovascular Disease Prevention and Management. Int J Clin Cardiol 4:103. doi.org/10.23937/2378-2951/1410103

Received: August 28, 2017; Accepted: September 25, 2017; Published: September 27, 2017

Copyright: (c) 2017 Yamaoka-Tojo M. This is an open-access article distributed under the terms of the Creative Commons Attribution License, which permits unrestricted use, distribution, and reproduction in any medium, provided the original author and source are credited. 


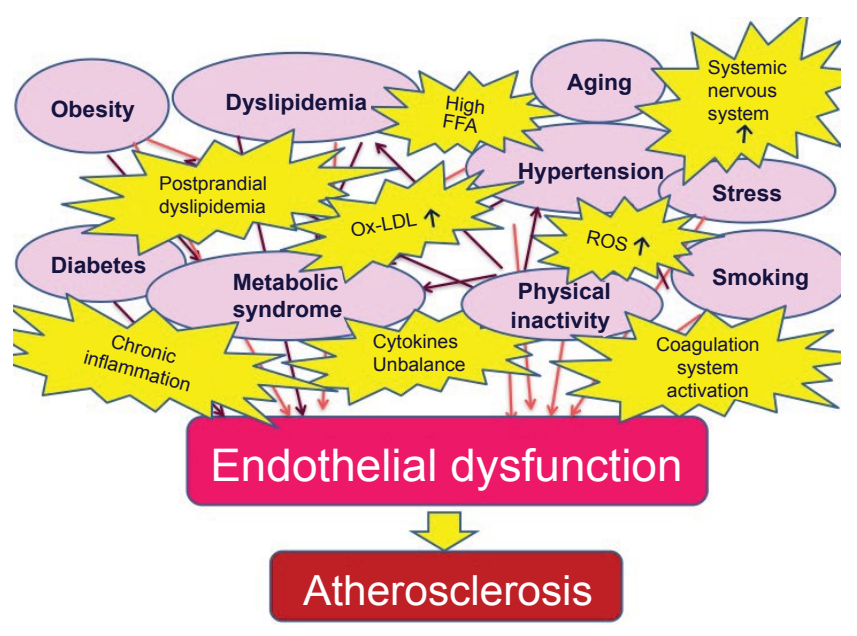

Figure 1: Endothelial function reflects atherosclerosis progression. Vascular endothelial function is decreased due to various factors such as smoking and physical inactivity, which are unfavorable for our health. Lifestyle diseases, such as hypertension, dyslipidemia, diabetes and obesity, induce vascular endothelial dysfunction. Vascular endothelial dysfunction is considered to have potential prognostic value for the early detection of cardiovascular disease.

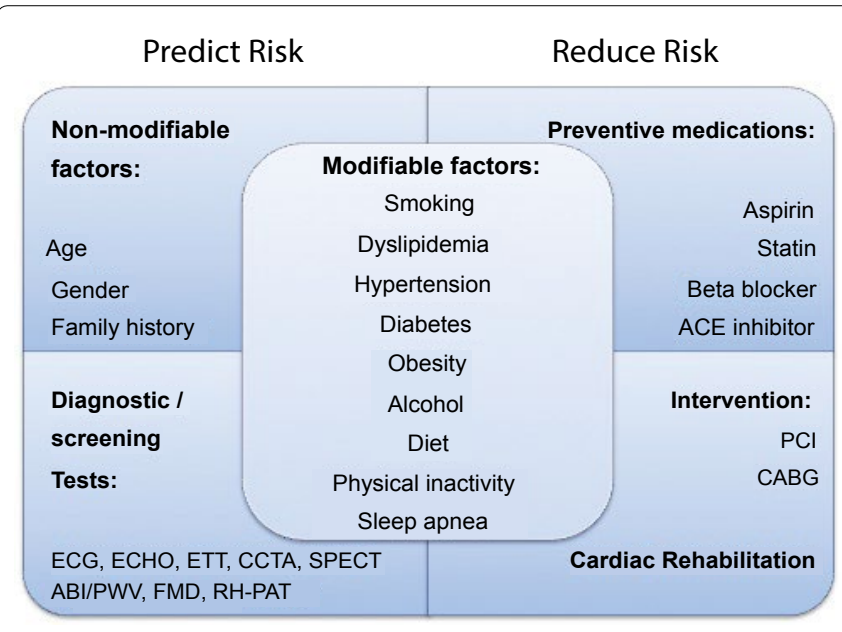

Figure 2: Cardiovascular disease prevention with total risk management. Risk factors can be divided into those that are useful in predicting risk and those that are useful targets for lowering risk.

ACE: Angiotensin-Converting Enzyme; PCl: Percutaneous Coronary Intervention; CABG: Coronary Artery Bypass Graft; ECG: Electrocardiography; ECHO: Echocardiography; ETT: Exercise Tolerance Test; CCTA: Coronary Computed Tomography Angiography; SPECT: Single Photon Emission Computed Tomography; ABI: Ankle Brachial Index; PWV: Pulse Wave Velocity; FMD: Flow-Mediated Dilation; RH-PAT: Reactive Hyperemia-Peripheral Arterial Tonometry.

ease progression is to reduce cardiac events and prevent chronic heart failure, and also improve their quality of life. Risk factors can be divided into those that are useful in predicting risk and those that are useful targets for lowering risk (Figure 2). For cardiovascular prevention and cardiovascular disease management to prevent secondary cardiac events, improving modifiable factors are very important. Among of them, favorable diet and increasing physical activities are critical modifiable risk factors.

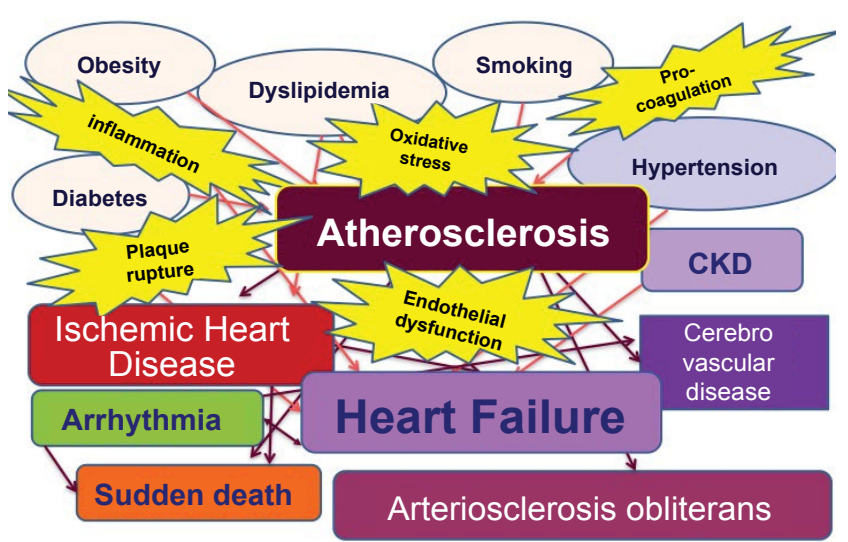

Figure 3: Risk factors for atherosclerosis progression and cardiovascular disease.

Basically, cardiovascular diseases are mainly based on atherosclerosis progression induced coronary risk factors, like as smoking, hypertension, dyslipidemia, diabetes mellitus, and obesity. With the progress of arteriosclerosis, ischemic heart disease develops, causing chronic heart failure as its terminal stage. Various mechanisms such as chronic inflammation, excessive oxidative stress, vascular endothelial dysfunction are involved in the development and progression of arteriosclerotic diseases in combination.

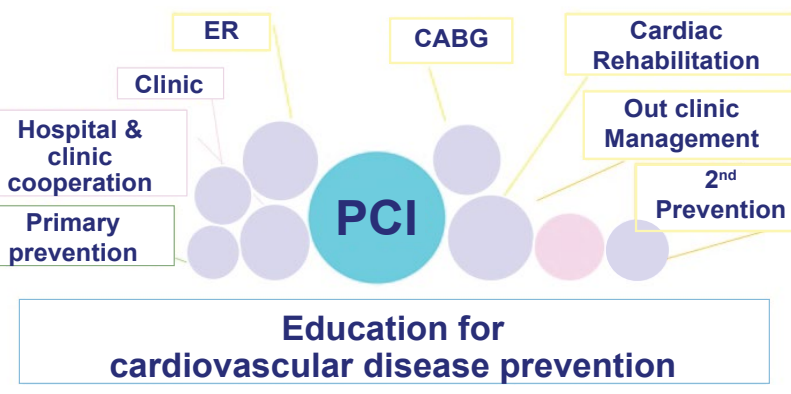

Figure 4: Total Disease Management for Cardiovascular Prevention.

In ischemic heart disease patients, cooperative total disease management is necessary to prevent cardiac events and heart failure progression. Even if medical doctors do special coronary interventions, it never prevent future cardiac events in patients with ischemic heart diseases without adequate disease management and patients' education. ER: Emergency Room; PCl: Percutaneous Coronary Intervention; CABG: Coronary Artery Bypass Grafting.

Basically, cardiovascular diseases are mainly based on atherosclerosis progression induced coronary risk factors, like as smoking, hypertension, dyslipidemia, diabetes, and obesity. These risk factors induce endothelial dysfunction, oxidative stress; coagulation cascade activation, inflammation and such a kind of morbidity stimulate atherosclerosis progression (Figure 3 ). To improve these risk factors, lifestyle change is known to be one of the main approaches for cardiovascular disease prevention.

For example, in ischemic heart disease patients, cooperative total disease management is necessary to prevent cardiac events and heart failure progression (Figure 4). There are a lot of important factors that we 
have to manage, like as, primary prevention, hospital and clinic cooperation, emergency room system, of course invasive therapy, Percutaneous Coronary Intervention $(\mathrm{PCl})$ and/or Coronary Artery Bypass Grafting (CABG), and also essential factors are cardiac rehabilitation and disease management for secondary prevention. In addition to these systemic and seamless disease management processes, patients' education for cardiovascular disease prevention plays an important role. Without disease management and patients' education, even if medical doctors do special coronary interventions, it never prevent future cardiac events in patients with ischemic heart diseases.

\section{What is Vascular Endothelial Function?}

Vascular endothelial dysfunction is a key event in the beginning of the development of atherosclerosis. Endothelial dysfunction is a predictor of stroke and heart diseases. Vascular endothelial function is considered to predict cardiac events in the future. According to a previous report, good endothelial function group has less cardiovascular events, compared to those of low endothelial function [11]. Normal functions of endothelial cells include mediation of coagulation, platelet adhesion, immune function and control of volume and electrolyte content of the intravascular and extravascular spaces.

What's wrong with endothelial dysfunction? Endothelial dysfunction is associated with reduced anticoagulant properties as well as increased adhesion molecule expression, chemokine and other cytokine release, as well as reactive oxygen species production from the endothelium. These lead to inflammation and fibroblast migration and proliferation inside the vessel wall, all of which play important roles in the development of atherosclerosis.

\section{Vascular Endothelial Function Measurement}

As one of the tool for visualization of patients' effort, we can measure vascular endothelial function. There are two non-invasive methods to measure cardiovascular function in clinical settings. Flow-Mediated Dilation (FMD) using Brachial Artery Ultrasound Imaging (BAUI) is more traditional testing for vascular function and already has a lot of evidence. On the other hand, Reactive Hyperemia-Pulse Amplitude Tonometry (RH-PAT) is a relatively new method of vascular endothelial function and still accumulating clinical evidence. Although \%FMD and RH-PAT index are considered to have a dominant positive correlation, it is thought that these two tests should not be the same because the principle of measurement method and the measurement site are different. Regarding the severity and complexity of coronary artery lesions, it was reported that vascular endothelial function measured by brachial artery FMD and RH-PAT in 80 patients with coronary artery disease [12]. In patients with multi-vessel disease or complex lesions, both
FMD and RH-PAT were significantly decreased and their predictive powers were almost the same. The authors concluded that RH-PAT could have fewer differences between examiners than FMD. In addition, according to a report of 115 hypertensive patients, RH-PAT reflected clearly the autonomic nervous activity by heart rate variability analysis compared to FMD [13]. From these results, FMD and RH-PAT should be regarded as seeing different responses even for the same vascular endothelial function test. Therefore, we need to understand the difference between the two tests and consider utilizing each of the advantages.

The reference value of $\%$ FMD is set to be $6 \%$ to $7 \%$ or more, and if it is less than $5 \%$, it is judged that there is a significant decrease in vascular endothelial function. Although it can be judged that there is obvious cardiovascular endothelial dysfunction at RH-PAT index of less than 1.5 , there are reports that it could be a significantly increase in cardiovascular events even if it is less than 1.70 [14].

Current measurements of endothelial function via FMD vary due to technical and physiological factors. A negative correlation between \%FMD and baseline artery size is recognized as a fundamental scaling problem, leading to biased estimates of endothelial function [15]. Another challenge of FMD is variability across centers and the requirement of highly qualified technicians to perform the procedure.

A non-invasive, FDA-approved device for measuring vascular endothelial function that work by measuring RH-PAT index is EndoPAT ${ }^{\circledR}$. RH-PAT index measured by EndoPAT ${ }^{\circledR}$ has shown an $80 \%$ sensitivity and $86 \%$ specificity to diagnose coronary artery spasm when compared against the gold standard, acetylcoline angiogram [16]. This result suggests that this peripheral test reflects the physiology of the coronary endothelium. It has been tested in several clinical trials at multiple centers. To detect vascular endothelial function, EndoPAT ${ }^{\circledR}$ is easy to use, non user-dependent, good reproducibility, and analyzes automatically $[17,18]$ (Figure 5). It was reported that $\mathrm{RH}$-PAT response depends on nitric oxide from vascular endothelium [19]. RH-PAT index relates to cardiovascular risk factors and predicts cardiovascular events $[18,20]$. Vascular endothelial function could be measured as an effective indicator for cardiovascular prevention in cardiac rehabilitation. RH-PAT has been tested in several clinical trials at multiple centers $[18,21,22]$. The results from clinical trials have shown that RH-PAT could be useful for risk evaluation, stratification and prognosis of getting major cardiovascular events $[18,20,23-26]$.

\section{Who is the Culprit to Induce Vascular Endothelial Dysfunction?}

Vascular endothelial dysfunction could be equal to vascular failure, which could not keep the balance of 

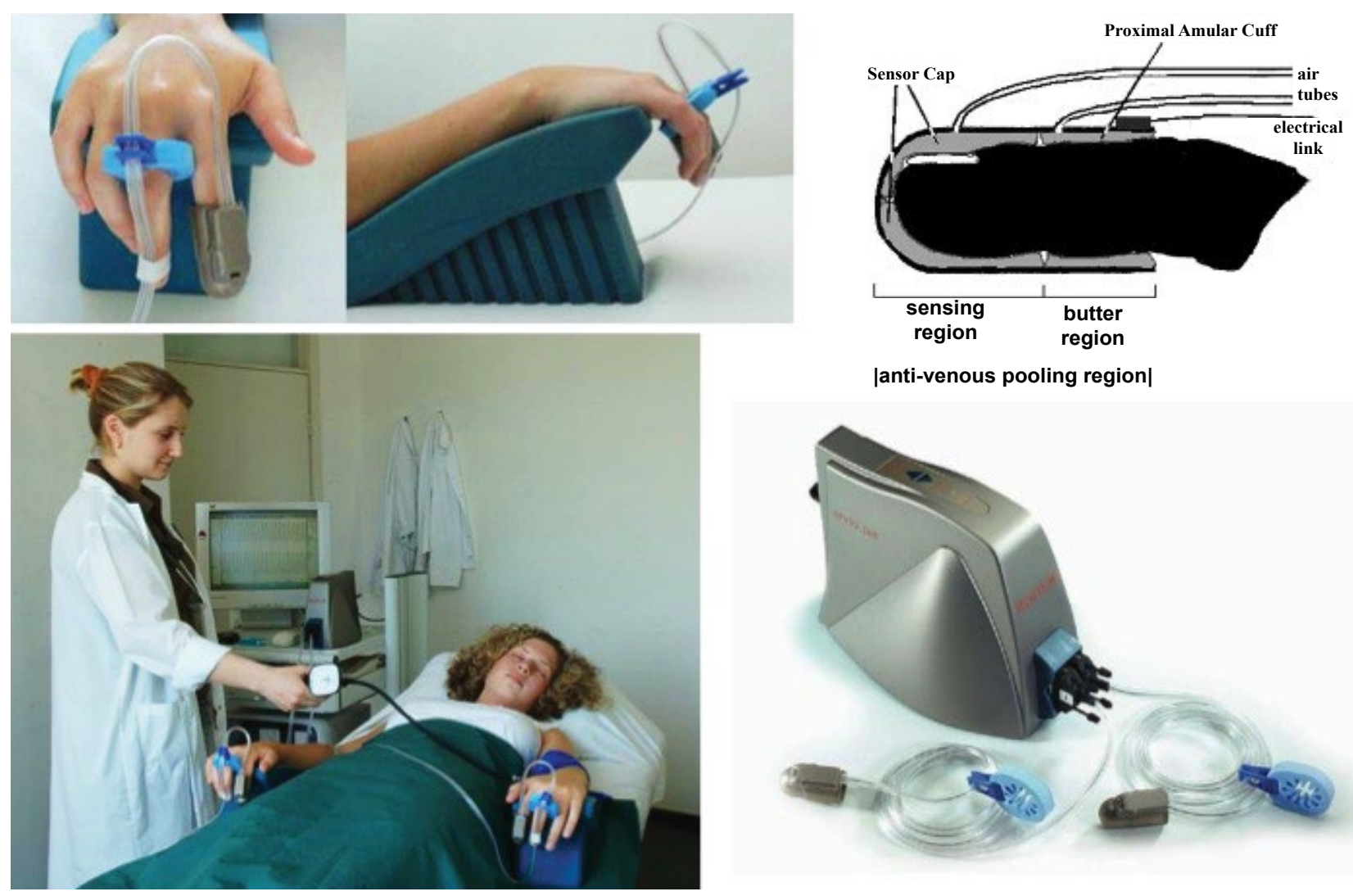

|anti-venous pooling region|

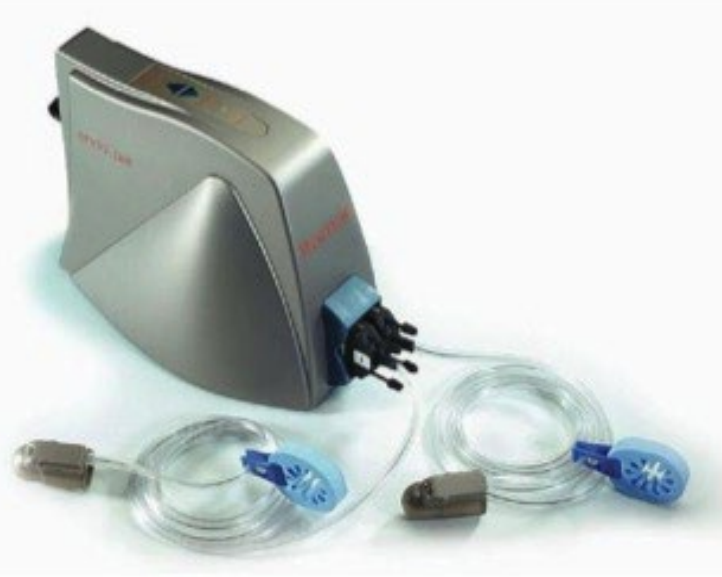

Figure 5: Endothelial function measured by EndoPAT ${ }^{\circledR}$.

EndoPAT ${ }^{\oplus}$ is used to measure especially microvascular endothelial function as an effective indicator of disease management for cardiovascular prevention [17].

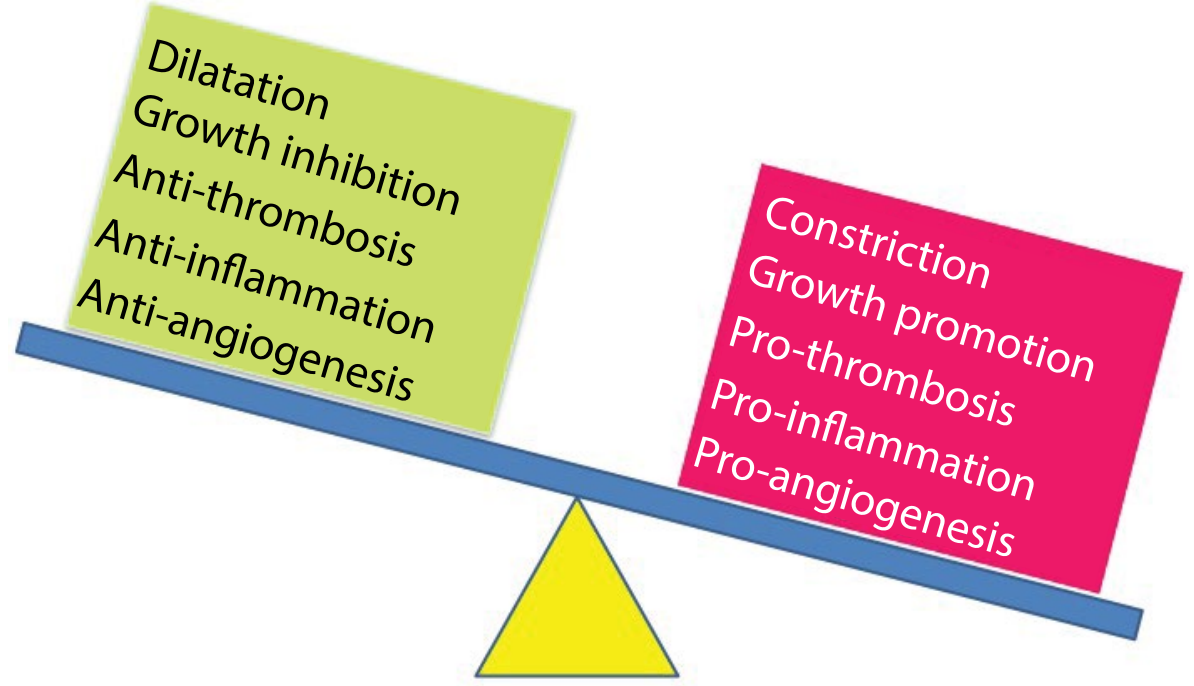

Figure 6: Endothelial dysfunction.

Endothelial dysfunction is equal to vascular failure, which cannot keep the balance of normal vascular conditions.

normal vascular conditions (Figure 6). Vascular endothelial dysfunction can result from and contribute to several disease processes, as occurs in hypertension, dyslipidemia, diabetes, collagen diseases like as Behcet's disease, septic shock, and it can also result from environmental factors, such as from smoking tobacco products and exposure to air pollution. Endothelial dysfunction is more prevalent in shift workers, a group known to have a higher risk for cardiovascular diseases.
There are a lot of risk factors to induce endothelial function in our daily life (Figure 7).

Why does endothelial dysfunction occur in patients with hypertension, dyslipidemia, diabetes mellitus, and/or obesity? It was reported that excess visceral adipose tissue worsens the vascular endothelial function measured by RH-PAT in type 2 diabetes mellitus and postprandial hyperglycemia $[27,28]$. On the other hand, measurement of endothelial function may prove critical to assess which 


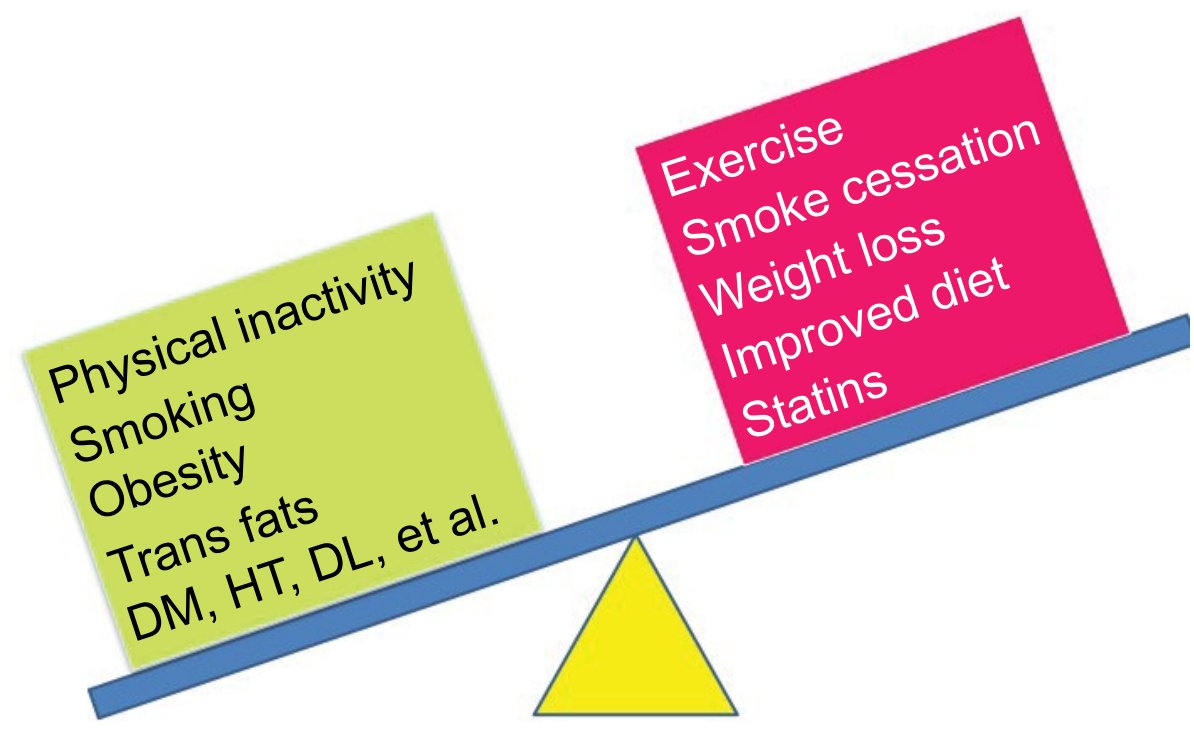

Figure 7: Risk and benefit factors for vascular endothelial function.

Vascular endothelial function could be critical to assess which sets of patients are improving their endothelial function. DM:

Diabetes Mellitus; HT: Hypertension; DL: Dyslipidemia.

sets of patients are improving their endothelial function. For example, a positive relationship exists between the consumption of trans fats (commonly found in hydrogenated products such as margarine and shortening) and the development of endothelial dysfunction.

\section{How Can We Improve Our Vascular Endothelial Function?}

So, what should we do to improve endothelial function? Endothelial function can be improved significantly by exercise, smoke cessation, weight loss in overweight or obese persons, and improved diet (Figure 7). For example, the Mediterranean diet, which consists of mono saturated fats from olive oil, pasta, fruits, vegetables, fish, whole grains, legumes/nuts, and moderate alcohol consumption, is considered to be good for our health. In the meal mainly using olive oil, FMD of 3 hours after meal was significantly lower than that of meal using canola oil or salmon oil, and the blood triglyceride levels and \%FMD had a negative correlation [29]. Because too much oxidized cholesterol induced by overheating decreases vascular endothelial function, there is a high possibility that omega-9 rich oleic acid, which is less oxidized even when heated, and less heated Mediterranean diet may be good factors to preserve favorable conditions in vascular endothelial function. When omega-3 unsaturated fatty acid was given to patients with a family history of diabetes for 12 weeks, vascular endothelial function measured by FMD was significantly improved ( $9.1 \pm 5.8 \%$ vs. $11.7 \pm 4.4 \%, P=0.02)$ and accompanied by significantly decreasing blood triglyceride levels and inflammatory biomarkers [30]. In the meta-analysis based on 16 studies, it has been found that omega- 3 unsaturated fatty acids increased FMD by $2.30 \%$ (95\% Cl: 0.89-3.72\%, $\mathrm{P}=0.001)$, at a dose ranging from 0.45 to $4.5 \mathrm{~g} / \mathrm{d}$ over a median of 56 days [31].
Coffee polyphone is expected to have anti-arteriosclerotic effect. It has been reported that vascular endothelial function measured by RH-PAT was significantly improved by coffee polyphenol intake in the $75 \mathrm{~g}$ oral glucose tolerance test [32]. On the other hand, it is revealed that vascular endothelial function measured by FMD decreased as an acute effect when caffeinated coffee were given compared with decaffeinated coffee [33]. However, it has been reported that \%FMD improved dose-dependently in decaffeinated espresso coffee [34]. In the case of ingesting in the long term, it seems preferable to take coffee rich in coffee polyphenols and avoid excessive intake of caffeine that causes increasing blood pressure and excessive activation of the sympathetic nerve system.

Treatment of hypertension and hypercholesterolemia are also critical; the major pharmacological interventions to improve endothelial function in those set of patients are statins, and renin angiotensin system inhibitors, (such as ACE inhibitors and angiotensin II receptor antagonists). Therefore, endothelial function testing might have potential prognostic value for the early detection of cardiovascular disease (Figure 1). Clinical trials in the recent years have demonstrated the feasibility of translating this measurement to the clinical practice in prevention for atherosclerosis progression. Statins have major pleiotropic anti-inflammatory and anti-oxidative effects besides the cholesterol reduction effect. These immune-modulatory effects of statins may explain why some patients improve their endothelial function with those drugs.

\section{Vascular Endothelial Glycocalyx}

According to previous papers, a protective gel-like coating layer of vascular endothelium, vascular endothelial glycocalyx is impaired in conditions such as Dia- 


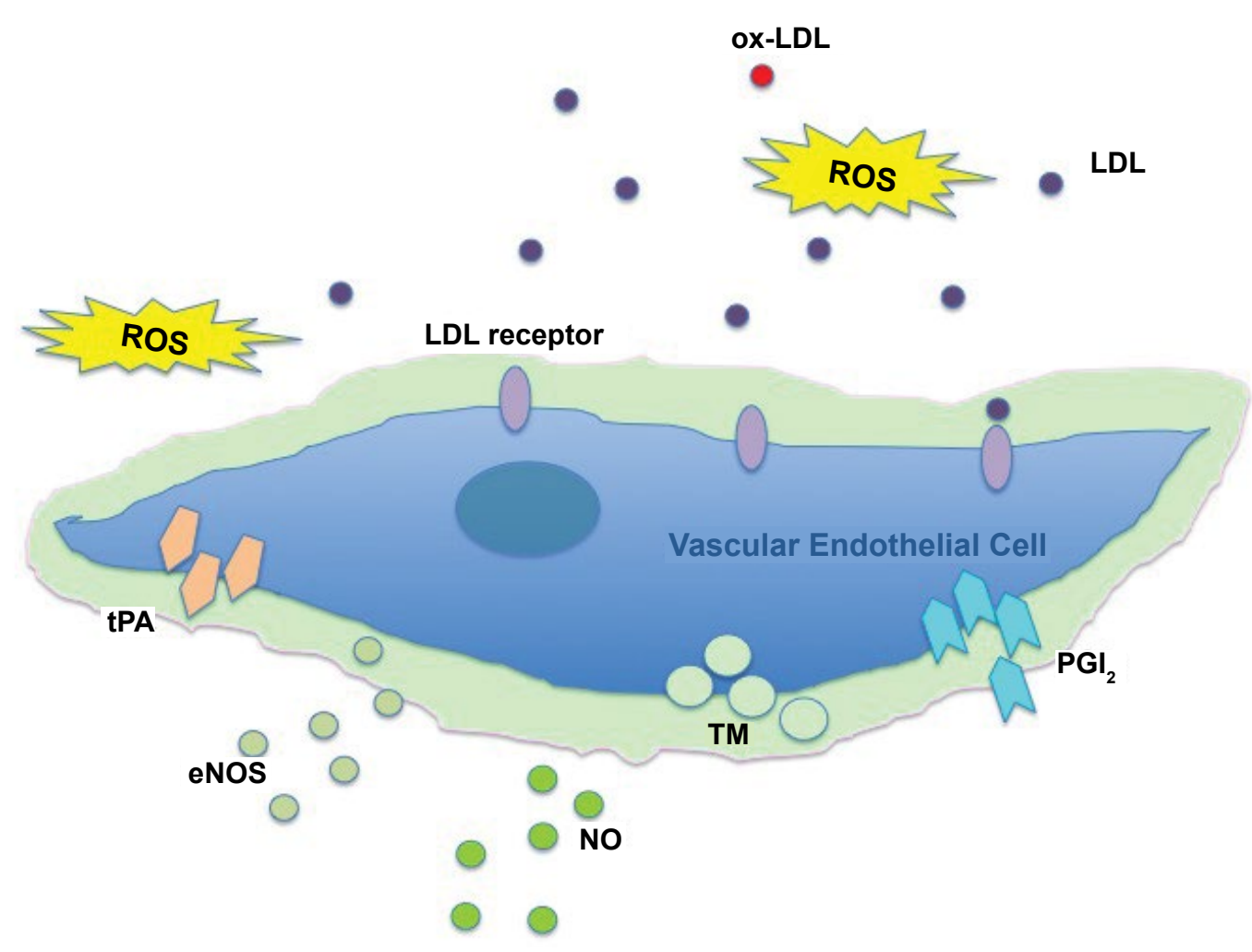

Figure 8: Healthy vascular endothelial glycocalyx.

ROS: Reactive Oxygen Species; NO: Nitric Oxide; eNOS: endothelial NO Synthase; TM: Thrombomodulin; tPA: tissue Plasminogen Activator; PGI2: Prostacyclin.

betes Mellitus (DM), dyslipidemia, and Chronic Kidney Disease (CKD) [35]. Glycocalyx is also an important regulator of endothelial cell-cell junctions. It was reported that widespread loss of the endothelial glycocalyx links albuminuric kidney disease with systemic vascular dysfunction [36]. Moreover, glycocalyx degradation instantly results in notable myocardial tissue edema [37].

What's the role of vascular glycocalyx? Vascular endothelial glycocalyx forms the interface between the vessel wall and the bloodstream, maintains the colloid osmotic gradient of the vascular barrier, and acts as a barrier to cell adhesion, plasma protein and water retention. In addition, vascular endothelial glycocalyx provides binding sites for Antithrombin III (AT III), tissue factor pathway inhibitors, lipoprotein lipase, Vascular Endothelial Growth Factor (VEGF), Fibroblast Growth Factor (FGF), extracellular Superoxide Dismutase (ecSOD), and hyaluronic acid molecules. It is thought that vascular endothelial glycocalyx acts as a shear stress sensor [38]. Healthy glycocalyx under physiological condition is considered to increase Nitric Oxide (NO) synthesis and protect endothelial cells from ROS and other inflammatory factors in blood stream (Figure 8). Vascular endothelial cells coated by the glycocalyx could play roles sufficiently and it may improve vascular endothelial function. Therefore, we hypothesize that one of the key regulator of vascular endothelial function is vascular glycocalyx [39].

Vascular endothelial glycocalyx is easily damaged by oxidized-low density lipoprotein [40]. Other damaging factors are high glucose, excessive low glucose, and inflammations, which damage and perturb be vascular endothelial glycocalyx. Vascular endothelial glycocalyx damage is associated with vascular endothelial dysfunction, which induces reduced NO bioavailability, increased excessive ROS production, inflammatory cytokine release, platelet adherence, coagulation, and leukocyte adhesion (Figure 9). Cell adhesion molecules are over-expressed in glycocalyx-damaged vascular endothelial cells. Then, directly attached leukocytes on vascular endothelial cell surface are easily migrated in the vascular wall in damaged glycocalyx-induced loose of cell-cell junctions. It's one of the initial steps of the development of atherosclerosis. Unfortunately, we cannot see this image in living person. So we measure endothelial function as an indirect indicator for vascular endothelial glycocalyx.

\section{Vascular Endothelial Function as an Indicator for Disease Management}

We use RH-PAT to measure especially microvascular endothelial function as an effective indicator of disease management for cardiovascular prevention. In ischemic heart disease patients, 4-week exercise intervention in cardiac rehabilitation significantly improved RH-PAT index in those patients (Figure 10).

Ezetimibe is a cholesterol lowering drug to inhibit cholesterol absorption from intestine for patients with hypercholesterolemia. Six-month Ezetimibe monother- 


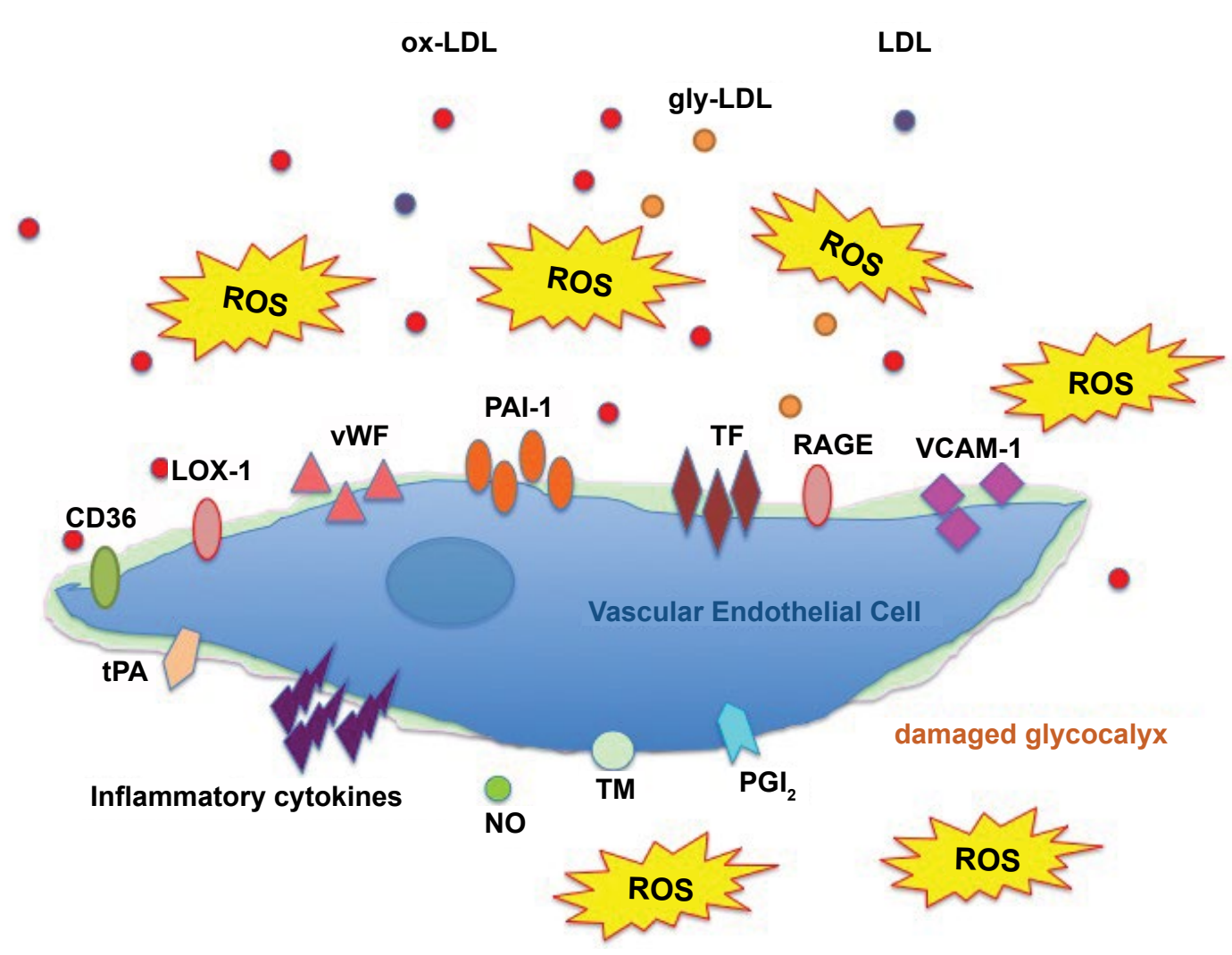

Figure 9: Damaged vascular endothelial glycocalyx.

Vascular endothelial glycocalyx damage is associated with vascular endothelial dysfunction, which induces reduced NO bioavailability, increased excessive ROS production, inflammatory cytokine release, platelet adherence, coagulation, and leukocyte adhesion.

NO: Nitric Oxide; ROS: Reactive Oxygen Species; PAI-1: Plasminogen Activator Inhibitor-1; TF: Tissue Factor; vWF: von Willebland Factor; RAGE: Receptor of Advanced Glycation End Product; LOX-1: Lectin-Like Oxidized LDL Receptor-1; tPA: tissue Plasminogen Activator; PGI2: Prostacyclin; TM: Thrombomodulin.

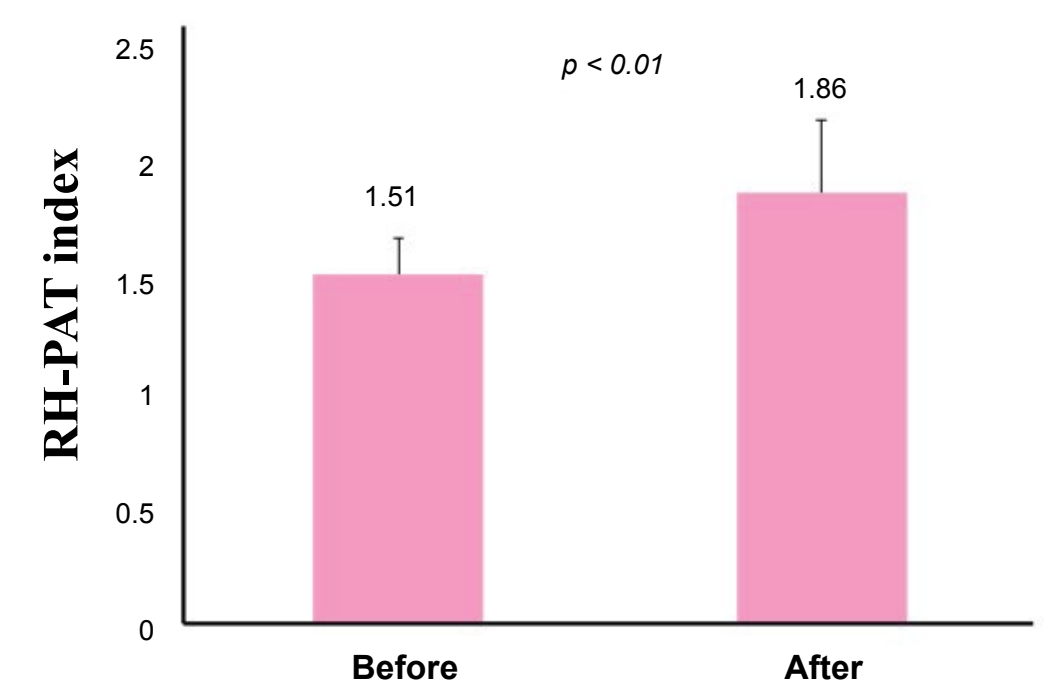

Figure 10: Exercise improved vascular endothelial function.

Four-week exercise with 30-minute walk per day significantly improved RH-PAT index $(n=27)$.

apy improved ET function by $15 \%$ increase [41]. When we added ezetimibe in dyslipidemia patients treated with atorvastatin, ezetimibe add-on therapy with atorvastatin improved endothelial function, too [42]. OxLDL is a strong inducer of vascular endothelial function; therefore the cholesterol absorbance inhibitor could be improving vascular endothelial function.
Vascular endothelial function is improved by a DPP-4 inhibitor, teneligliptin, in patients with DM. Three-month teneligliptin administration significantly improved vascular endothelial function, which was related to improvement of $\mathrm{HbA1c}$ from 7.6 to $6.9 \%$ [43]. In diabetes patients, LV diastolic dysfunction is an important risk factor to induce heat failure progression. 3-month teneligliptin administration 
improves LV diastolic function in almost half of our study patients. The improvement of RH-PAT index measured by EndoPAT is significantly associated with decrease of $E$ over e prime (E/e'), which means improvement of LV diastolic function. It suggests that the therapy may improve LV diastolic function associated with microvascular endothelial function improvement in patients with diabetes.

\section{Vascular Endothelial Function and Cardiovas- cular Risks}

According to a previous paper about EndoPAT in Framingham Third Generation Cohort participants, $\mathrm{RH}$ PAT index was related to multiple traditional and metabolic cardiovascular risk factors [18]. Their findings support further investigations to define the clinical utility and predictive value of digital pulse amplitude. Furthermore, Dr. Rubinstein, et al. has been shown that RH-PAT predicts cardiovascular adverse events, a combination of cardiac death/myocardial infarction/coronary revascularization and cardiac hospitalizations in patients with low Reactive Hyperemia Index (RHI) [20]. Their cut off ratio of the natural Logarithmically-scaled LRHI (L_RHI) is 0.4. RH-PAT was reported to add incremental predictive ability to traditional risk factors for prognosis of CAD patients successfully treated with statins [44].

\section{Vascular Endothelial Function and Coronary Artery Disease}

Dr. Matsuzawa, et al. reported that RH-PAT index predicts the presence of Ischemic Heart Disease (IHD), especially non-obstructive coronary artery disease [24]. Vascular endothelial dysfunction was found in half of women with chest pain, in the absence of overt blockages in large coronary arteries, termed as coronary microvascular dysfunction. In addition, the authors published another data about EndoPAT, in which RH-PAT index predicts cardiovascular events [14]. Advanced endothelial dysfunction was significantly correlated with near future cardiovascular events in high-risk patients. Kaplan-Meier analysis for the probability of cardiovascular events in CAD patients based on median value was $L_{-}$RHI 0.501. This physiological vascular measurement improved risk discrimination when added to the Framingham risk score, BNP, and the Synergy between $\mathrm{PCl}$ with Taxus and Cardiac Surgery score (SYNTAXsc). According to a recent paper, vascular endothelial function measured by RH-PAT is an independent predictor of 1-year adverse clinical outcome in coronary atherosclerosis patients hospitalized in the emergency department Chest Pain Unit (CPU) [45]. Regarding the 20 patients who developed Major Adverse Cardiovascular End-point (MACE) out of consecutive 300 subjects, RHI $\leq$ the median was associated with higher 1-year MACE ( $13 \%$ vs. $0.7 \%, \mathrm{P}<0.001$ ) compared to $\mathrm{RHI}>$ the median. Multivariate analysis demonstrated that $\mathrm{RHI} \leq$ the median is an independent predictor of coronary atherosclerosis lesions in the CPU patients underwent coronary computerized tomographic angiography (OR 5.98, 95\% $\mathrm{Cl}$ 03.29-10.88; P < 0.001) and 1-year MACE (OR 15.207, 95\% Cl 2.00-115.33; P < 0.01). RH-PAT index may have clinical utility in triaging patients in the CPU and in predict 1-year MACE.

\section{Vascular Endothelial Function and Heart Failure}

In Japan and many other developed countries, the rapid increase in chronic heart failure is becoming a social problem accompanied by the increase number of aged people. The proportion of Heart Failure with preserved left ventricular Ejection Fraction (HFpEF) patients among patients with heart failure increases with age [46]. Although the cause of HFpEF is not completely elucidated, it may be caused by vascular endothelial dysfunction of microvessels in the heart [47].

\section{Vascular Endothelial Function and Thrombosis}

Virchow's triad consists of blood flow stagnation, hypercoagulability, and vascular endothelial dysfunction, and is known as risk factors for Venous Thromboembolism (VTE). As well as vascular endothelial dysfunction, blood flow stagnation reduces vascular endothelial function by decreasing shear stress, and $\mathrm{PGI}_{2}$ (Prostacyclin) production reduction by vascular endothelial dysfunction promotes platelet aggregation and blood coagulation. In other words, constituent factors of Virchow's triad are the result of vascular endothelial dysfunction. From such a viewpoint, vascular endothelial function test is considered to be useful for risk stratification and prognostic prediction of onset of Deep Vein Thrombosis (DVT) and intra-atrial thrombus formation in atrial fibrillation [48]. Vascular endothelial glycocalyx-targeting therapeutics is considered to be useful to detect antithrombogenic capacity and treat damaged vasculatures [49].

\section{Cardiac Rehabilitation and Vascular Endothelial Function}

Cardiac rehabilitation is a program designed for cardiovascular disease prevention and treatment. It includes physical exercise, lifestyle changes, education and emotional support. There are 3 phases in cardiac rehabilitation. Phase I, acute phase of cardiac rehabilitation, is fully recognized in its usefulness to prevent cardiovascular disease recurrence and improve Patient's Quality of Life (QOL). Instead of this, phase II to III, chronic phase (intermediate to maintenance phase) of cardiac rehabilitation, is not common in many countries. Because of medical budget limitation and difficulty to evaluate their efficacy. In such a situation, how to evaluate the efficacy of cardiac rehabilitation in chronic phase, especially as a short-term evaluation? For patients, visualization of their effort or lifestyle change is essential to keep their motivation to prevent cardiovascular disease progression. Vascular endothelial function is considered to predict cardiac events in the future. We use RH-PAT to measure vascular endothelial function as an ef- 
fective indicator of disease management, including cardiac rehabilitation. In regard to stretching exercises, RH-PAT index was significantly higher immediately after the exercise than before, which was accompanied by increasing of high-frequency components $(\mathrm{HF}, 0.15-0.40 \mathrm{~Hz}$ ) of heart rate variability as an indicator of parasympathetic nervous activities, and transcutaneous Oxygen Pressure $\left(\mathrm{tcPO} \mathrm{C}_{2}\right)$ on the right Foot and Chest ratio (Foot-tcPO ${ }_{2} /$ Chest-tcPO ${ }_{2}$ ) [50]. Cardiac rehabilitation with 4-week stretching exercises induced a significant increase in the RH-PAT index and significant decreases in serum levels of von Willebland Factor (vWF), Malondialdehyde-modified Low-Density Lipoprotein cholesterol (MDA-LDL), reactive oxygen species, and fibrinogen concentrations [51]. Cardiac rehabilitation exercise training program of moderate intensity ( 3 session/ week on a bicycle at $60-70 \%$ of Heart Rate Reserve (HRR) for 3 months, followed by one session/week until 1-year follow-up) improved cardiopulmonary function measured by cardiopulmonary exercise stress test and endothelial function measured by RH-PAT in women in breast cancer [52]. In contrast, a 12-week supervised cardiac rehabilitation program with 3 sessions per week at an intensity of $80 \%$ of HRR, improved endothelial function assessed by FMD but not by RH-PAT in patients with coronary heart disease [53]. In general, effects of aerobic exercise can be obtained with $40-60 \%$ of HRR, and effect of anaerobic exercise is primarily obtained at $60 \%$ of HRR and above. Since exercise intensity of cardiac rehabilitation usually aims at 40-60\% of HRR, anaerobic exercise effects might be examined mainly in the study.

\section{Conclusions}

Vascular endothelial function may be useful indicators in primary prevention of cardiovascular diseases, especially in participants who has accumulating coronary risk factors. Since cardiovascular endothelial dysfunction is also detected in patients with heart failure, it is expected to play an important role as a prognostic predictor for cardiovascular events. Moreover, vascular endothelial function could be a target for comprehensive disease management for cardiovascular disease prevention. "A man is as old as his arteries" (William Osler: 1849-1919), vascular endothelial function is an integrated index showing the health of blood vessels, and keeping the vascular endothelial function in good condition leads to the health of the living body.

\section{References}

1. Ross R (1999) Atherosclerosis-an inflammatory disease. $N$ Engl J Med 340: 115-126.

2. Widlansky ME, Gokce N, Keaney JF Jr, Vita JA (2003) The clinical implications of endothelial dysfunction. J Am Coll Cardiol 42: 1149-1160.

3. Suwaidi JA, Hamasaki S, Higano ST, Nishimura RA, Holmes DR Jr, et al. (2000) Long-term follow-up of patients with mild coronary artery disease and endothelial dysfunction. Circulation 101: 948-954.

4. Heitzer T, Schlinzig T, Krohn K, Meinertz T, Munzel T
(2001) Endothelial dysfunction, oxidative stress, and risk of cardiovascular events in patients with coronary artery disease. Circulation 104: 2673-2678.

5. Hasdai D, Gibbons RJ, Holmes DR Jr, Higano ST, Lerman A (1997) Coronary endothelial dysfunction in humans is associated with myocardial perfusion defects. Circulation 96: 3390-3395.

6. Zeiher AM, Krause T, Schachinger V, Minners J, Moser E (1995) Impaired endothelium-dependent vasodilation of coronary resistance vessels is associated with exercise-induced myocardial ischemia. Circulation 91: 2345-2352.

7. Lerman A, Zeiher AM (2005) Endothelial function: Cardiac events. Circulation 111: 363-368.

8. Targonski PV, Bonetti PO, Pumper GM, Higano ST, Holmes DR Jr, et al. (2003) Coronary endothelial dysfunction is associated with an increased risk of cerebrovascular events. Circulation 107: 2805-2809.

9. Schachinger V, Britten MB, Zeiher AM (2000) Prognostic impact of coronary vasodilator dysfunction on adverse long-term outcome of coronary heart disease. Circulation 101: 1899-1906.

10. Halcox JP, Schenke WH, Zalos G, Mincemoyer R, Prasad A, et al. (2002) Prognostic value of coronary vascular endothelial dysfunction. Circulation 106: 653-658.

11. Yeboah J, Folsom AR, Burke GL, Johnson C, Polak JF, et al. (2009) Predictive value of brachial flow-mediated dilation for incident cardiovascular events in a population-based study: The multi-ethnic study of atherosclerosis. Circulation 120: 502-509.

12. Woo JS, Jang WS, Kim HS, Lee JH, Choi EY, et al. (2014) Comparison of peripheral arterial tonometry and flow-mediated vasodilation for assessment of the severity and complexity of coronary artery disease. Coron Artery Dis 25: 421-426.

13. Tomiyama H, Yoshida M, Higashi $Y$, Takase B, Furumoto $T$, et al. (2014) Autonomic nervous activation triggered during induction of reactive hyperemia exerts a greater influence on the measured reactive hyperemia index by peripheral arterial tonometry than on flow-mediated vasodilatation of the brachial artery in patients with hypertension. Hypertens Res 37: 914-918.

14. Matsuzawa $Y$, Sugiyama $S$, Sumida H, Sugamura K, Noza$\mathrm{ki}$, et al. (2013) Peripheral endothelial function and cardiovascular events in high-risk patients. J Am Heart Assoc 2: e000426.

15. Maruhashi T, Soga J, Fujimura N, Idei N, Mikami S, et al. (2013) Relationship between flow-mediated vasodilation and cardiovascular risk factors in a large community-based study. Heart 99: 1837-1842.

16. Bonetti PO, Pumper GM, Higano ST, Holmes DR Jr, Kuvin JT, et al. (2004) Noninvasive identification of patients with early coronary atherosclerosis by assessment of digital reactive hyperemia. J Am Coll Cardiol 44: 2137-2141.

17. Celermajer DS (2008) Reliable endothelial function testing: At our fingertips? Circulation 117: 2428-2430.

18. Hamburg NM, Keyes MJ, Larson MG, Vasan RS, Schnabel $\mathrm{R}$, et al. (2008) Cross-sectional relations of digital vascular function to cardiovascular risk factors in the Framingham Heart Study. Circulation 117: 2467-2474.

19. Nohria A, Gerhard-Herman M, Creager MA, Hurley S, Mitra $D(2006)$ Role of nitric oxide in the regulation of digital pulse volume amplitude in humans. J Appl Physiol 101: 545-548. 
20. Rubinshtein R, Kuvin JT, Soffler M, Lennon RJ, Lavi S, et al. (2010) Assessment of endothelial function by non-invasive peripheral arterial tonometry predicts late cardiovascular adverse events. Eur Heart J 31: 1142-1148.

21. Mulukutla SR, Venkitachalam L, Bambs C, Kip KE, Aiyer A, et al. (2010) Black race is associated with digital artery endothelial dysfunction: Results from the Heart SCORE study. Eur Heart J 31: 2808-2815.

22. Schnabel RB, Wild PS, Schulz A, Zeller T, Sinning CR, et al. (2012) Multiple endothelial biomarkers and noninvasive vascular function in the general population: The Gutenberg Health Study. Hypertension 60: 288-295.

23. Kuvin JT, Patel AR, Sliney KA, Pandian NG, Sheffy J, et al. (2003) Assessment of peripheral vascular endothelial function with finger arterial pulse wave amplitude. Am Heart $\mathrm{J}$ 146: $168-174$

24. Matsuzawa Y, Sugiyama S, Sugamura K, Nozaki T, Ohba $\mathrm{K}$, et al. (2010) Digital assessment of endothelial function and ischemic heart disease in women. $\mathrm{J} \mathrm{Am}$ Coll Cardiol 55: 1688-1696.

25. Akiyama E, Sugiyama S, Matsuzawa $Y$, Konishi M, Suzuki $\mathrm{H}$, et al. (2012) Incremental prognostic significance of peripheral endothelial dysfunction in patients with heart failure with normal left ventricular ejection fraction. J Am Coll Cardiol 60: 1778-1786.

26. Matsue $Y$, Suzuki M, Nagahori W, Ohno M, Matsumura A, et al. (2013) Endothelial dysfunction measured by peripheral arterial tonometry predicts prognosis in patients with heart failure with preserved ejection fraction. Int J Cardiol 168: 36-40.

27. Kurozumi A, Okada Y, Arao T, Tanaka Y (2016) Excess visceral adipose tissue worsens the vascular endothelial function in patients with type 2 diabetes mellitus. Intern Med 55: 3091-3095.

28. Keiichi Torimoto, Yosuke Okada, Hiroko Mori, Yoshiya Tanaka (2014) Low levels of 1,5-anhydro-D-glucitol are associated with vascular endothelial dysfunction in type 2 diabetes. Cardiovasc Diabetol 13: 99.

29. Vogel RA, Corretti MC, Plotnick GD (2000) The postprandial effect of components of the Mediterranean diet on endothelial function. J Am Coll Cardiol 36: 1455-1460.

30. Rizza S, Tesauro M, Cardillo C, Galli A, lantorno M, et al. (2009) Fish oil supplementation improves endothelial function in normoglycemic offspring of patients with type 2 diabetes. Atherosclerosis 206: 569-574.

31. Wang Q, Liang X, Wang L, Lu X, Huang J, et al. (2012) Effect of omega-3 fatty acids supplementation on endothelial function: A meta-analysis of randomized controlled trials. Atherosclerosis 221: 536-543.

32. Ochiai R, Sugiura $Y$, Shioya $Y$, Otsuka K, Katsuragi $Y$, et al (2014) Coffee polyphenols improve peripheral endothelial function after glucose loading in healthy male adults. Nutr Res 34: 155-159.

33. Buscemi S, Verga S, Batsis JA, Donatelli M, Tranchina MR, et al. (2010) Acute effects of coffee on endothelial function in healthy subjects. Eur J Clin Nutr 64: 483-489.

34. Buscemi S, Verga S, Batsis JA, Tranchina MR, Belmonte $S$, et al. (2009) Dose-dependent effects of decaffeinated coffee on endothelial function in healthy subjects. Eur $\mathrm{J}$ Clin Nutr 63: 1200-1205.

35. Rutledge JC, Ng KF, Aung HH, Wilson DW (2010) Role of triglyceride-rich lipoproteins in diabetic nephropathy. Nat Rev Nephrol 6: 361-370.
36. Ueda A, Shimomura M, Ikeda M, Yamaguchi R, Tanishita K (2004) Effect of glycocalyx on shear-dependent albumin uptake in endothelial cells. Am J Physiol Heart Circ Physiol 287: 2287-2294.

37. Van den Berg BM, Vink H, Spaan JA (2003) The endothelial glycocalyx protects against myocardial edema. Circ Res 92: 592-594.

38. Akinori Ueda, Masaki Koga, Mariko Ikeda, Susumu Kudo, Kazuo Tanishita (2004) Effect of shear stress on microvessel network formation of endothelial cells with in vitro three-dimensional model. Am J Physiol Heart Circ Physiol 287: 994-1002.

39. Minako Yamaoka Tojo (2014) Cardiac rehabilitation-mediated molecular mechanisms of cardiovascular protection. Circ J 78: 2624-2626.

40. Vink H, Constantinescu AA, Spaan JA (2000) Oxidized lipoproteins degrade the endothelial surface layer : implications for platelet-endothelial cell adhesion. Circulation 101: 1500-1502.

41. Yamaoka-Tojo M, Tojo T, Takahira N, Masuda T, Izumi T (2011) Ezetimibe and reactive oxygen species. Curr Vasc Pharmacol 9: 109-120.

42. Minako Yamaoka-Tojo, Taiki Tojo, Rie Kosugi, Yuko Hatakeyama, Yuki Yoshida, et al. (2009) Effects of ezetimibe add-on therapy for high-risk patients with dyslipidemia. Lipids Health Dis 8: 41.

43. Hashikata T, Yamaoka-Tojo M, Kakizaki R, Nemoto T, Fujiyoshi K, et al. (2016) Teneligliptin improves left ventricular diastolic function and endothelial function in patients with diabetes. Heart Vessels 31: 1303-1310.

44. Matsue $Y$, Yoshida K, Nagahori W, Ohno M, Suzuki M, et al. (2014) Peripheral microvascular dysfunction predicts residual risk in coronary artery disease patients on statin therapy. Atherosclerosis 232: 186-190.

45. Shechter M, Matetzky S, Prasad M, Goitein O, Goldkorn R (2017) Endothelial function predicts 1-year adverse clinical outcome in patients hospitalized in the emergency department chest pain unit. Int J Cardiol 240: 14-19.

46. Tribouilloy C, Rusinaru D, Mahjoub H, Souliere V, Levy F, et al. (2008) Prognosis of heart failure with preserved ejection fraction: A 5 year prospective population-based study. Eur Heart J 29: 339-347.

47. Borlaug BA, Olson TP, Lam CS, Flood KS, Lerman A, et al. (2010) Global cardiovascular reserve dysfunction in heart failure with preserved ejection fraction. $\mathrm{J} \mathrm{Am}$ Coll Cardiol 56: $845-854$

48. Freestone B, Chong AY, Nuttall S, Lip GY (2008) Impaired flow mediated dilatation as evidence of endothelial dysfunction in chronic atrial fibrillation: relationship to plasma von Willebrand factor and soluble E-selectin levels. Thromb Res 122: 85-90.

49. Sashka Dimitrievska, Liqiong Gui, Amanda Weyers, Tylee Lin, Chao Cai, et al. (2016) New functional tools for antithrombogenic activity assessment of live surface glycocalyx. Arterioscler Thromb Vasc Biol 36: 1847-1853.

50. Hotta K, Kamiya K, Shimizu R, Yokoyama M, Nakamura-Ogura M, et al. (2013) Stretching exercises enhance vascular endothelial function and improve peripheral circulation in patients with acute myocardial infarction. Int Heart J 54: 59-63.

51. Kato M, Masuda T, Ogano M, Hotta K, Takagi $H$, et al. (2017) Stretching exercises improve vascular endothelial dysfunction through attenuation of oxidative stress in 
chronic heart failure patients with an implantable cardioverter defibrillator. J Cardiopulm Rehabil Prev 37: 130-138.

52. Giallauria F, Vitelli A, Maresca L, Santucci De Magistris M, Chiodini P, et al. (2016) Exercise training improves cardiopulmonary and endothelial function in women with breast cancer: Findings from the Diana-5 dietary intervention study. Intern Emerg Med 11: 183-189.
53. Cornelissen VA, Onkelinx S, Goetschalckx K, Thomaes T, Janssens S, et al. (2014) Exercise-based cardiac rehabilitation improves endothelial function assessed by flow-mediated dilation but not by pulse amplitude tonometry. Eur $\mathrm{J}$ Prev Cardiol 21: 39-48. 